\title{
The Nature of Accretion in Seyfert Galaxies
}

\author{
Nwankwo Ifeanyi Francis, Ogwo Jemima Ngozi \\ Department of Physics, Abia State University, Uturu, Nigeria \\ Email address: \\ ifeanyichy@gmail.com (N. I. Francis),jimogwo@yahoo.com (O. J. Ngozi)
}

To cite this article:

Nwankwo Ifeanyi Francis, Ogwo Jemima Ngozi. The Nature of Accretion in Seyfert Galaxies. International Journal of Astrophysics and Space Science. Vol. 9, No. 2, 2021, pp. 32-36. doi: 10.11648/j.ijass.20210902.12

Received: June 22, 2021; Accepted: July 7, 2021; Published: July 27, 2021

\begin{abstract}
We present statistical results from a sample of 91,006 Seyfert galaxies obtained from SDSS DR14 (Sloan Digital Sky Survey Data Release 14). Using the ratio of the flux of doubly ionized oxygen [OIII] to that of the flux of hydrogen beta line $H_{\beta}$, the Seyfert galaxy sample was separated into Seyferts 1 and 2. For each class of Seyfert galaxy, both bolometric luminosity and Eddington luminosity were calculated and these results were used to investigate the nature of accretion in both classes by estimating their Eddington ratios. The susceptibility of the [OIII] line to reddening necessitated its being extinction corrected before calculating bolometric luminosity from $L_{b o l}=3500 L_{[O I I]}$. Our work shows that Seyfert 2 galaxies are 10 times more luminous than their Seyfert 1 counterparts. We also found Seyfert 2 galaxies to be centrally more massive than Seyfert 1 galaxies as a result of which Eddington ratio is less in Seyfert 1 galaxies. These results show that both Seyferts 1 and 2 galaxies are powered by thick accretion disk and as such undergo predominantly super-Eddington accretion. Only a negligible percent of them $(0.49 \%$ for Seyfert 1 galaxies and $0.28 \%$ for Seyfert 2 galaxies) are powered by thin accretion disk. Our results also indicate that black hole growth in both Seyfert 1 and Seyfert 2 galaxies is dominated by super-Eddington accretion rather than sub-Eddington accretion showing that though both Seyfert classes are good accretors, Seyfert 2 galaxies are better accretors and this means that Seyferts of thin accretion disks are still in the low-Eddington accretion era.
\end{abstract}

Keywords: Blackhole, Eddington-luminosity, Bolometric-luminosity, Eddington-ratio, Reddening, Accretion, Narrow Line Region, Broad Line Region, Extended Narrow Line Region

\section{Introduction}

Seyfert galaxies are active galactic nuclei powered by super massive black holes (SMBHs). Though they emit radiations across all spectra, they are more luminous in the optical wavelength and this has given then their identity. The presence of SMBHs in their nuclei makes their centers bright. Majority of them are spiral and are associated with star formation. They have spectra with strong emission lines that reveal both narrow and broad lines believed to come from illuminated gas clouds by the central engine. Seyfert galaxies have two subclasses named as Seyfert 1 and Seyfert 2 based on the presence and/or absence of broad emission lines in their spectra [1]. Seyfert 1 galaxies have both broad emission lines and narrow emission lines while Seyfert 2 galaxies have only narrow emission lines [11].

Notably, Seyfert galaxies have bright star-like nuclei whose spectra show emission lines covering a wide range of ionization stages. The major contents of a Seyfert nucleus are a central source, the broad line region (BLR), a molecular dusty torus, the narrow line region (NLR) and a possible extended narrow line region (ENLR). The broad line region (BLR) is characterized by high density clouds much greater than $10^{6} \mathrm{~cm}^{-3}$ and close to the central region where, it is believed, they are Doppler boosted. The broad line region (BLR) has a typical sub parsec size surrounded by an optically thick torus of dust [12]. The narrow line region (NLR) is characterized by low-density clouds $\left(\mathrm{Ne} \sim 10^{2}\right.$ $10^{6} \mathrm{~cm}^{-3}$ ), temperature $\mathrm{T} \sim 10^{4} \mathrm{~K}$ and full-width at halfmaximum (FWHM) of emission lines in the range $\sim 200-$ $1000 \mathrm{kms}$ [5]. The confined ionizing radiation in the cones ionizes gas clouds by a non-thermal power-law spectrum from the central region [6]. The extended narrow line region (ENLR) is a region of highly ionized gas of size between $15 \mathrm{pc}$ and $20 \mathrm{kpc}$. Because of the proximity of the ENLR to the extremely hot nucleus, it is associated with photo ionization and shocks as a result atoms within it are usually ionized giving rise to high concentration of plasma.

In this work, we use a large sample $(91,006)$ of Seyfert 
galaxies to study the nature of accretion in Seyfert galaxies with the intent to determine which of the two classes of Seyfert galaxies is a better accretor and centrally more energetic.

\section{Data Sample}

This study is based on a sample of 91,006 Seyfert galaxies from the Sloan Digital Sky Survey (SDSS)-14. Some of the parameters calculated from the data made available by SDSS-14 with which we did this study include Bolometric luminosity (using extinction corrected expression), Eddington luminosity, Eddington ratio, Reddening and Blackhole mass. Using the line flux criterion of $[\mathrm{OIII}] / \mathrm{H}_{\beta}>3$, [9] for Seyfert 2 galaxies, the 91,006 Seyfert galaxies published by SDSS-DR14 were separated into Seyfert 1 galaxies and Seyfert 2 galaxies which produced 7,859 Seyfert 1 galaxies and 83,147 Seyfert 2 galaxies.

\section{Reddening in Seyfert Galaxies}

In the nucleus of galaxies are dusts which cause absorption and scattering of radiation depending on their wavelengths. Radiation of shorter wavelengths are absorbed and/or scattered more than the longer wavelengths whose wavelengths literally are bigger than the dust particles and as such are neither scattered nor absorbed. However, when radiations whose wavelengths are comparable to the dust sizes are absorbed, the particles of the dust absorbing these radiations get heated up and emit radiation usually in the near and far infrared energy spectra and this tends to contaminate the Balmer lines emitted from HII regions of star forming galaxies making its energy spectra appear red. This contamination effect is called reddening which is determined by the Balmer decrement that is the ratio of the flux of hydrogen alpha $(\mathrm{H} \alpha)$ to that of hydrogen Beta $\left(\mathrm{H}_{\beta}\right)$ and it is used to examine and predict the presence and/or absence of torus in each of the objects. Balmer decrement $\left(\frac{H_{\alpha}}{H_{\beta}}\right)=2.86$ or less means that there is no reddening. But if it is more than 2.86, it signifies the presence of reddening (Osterbrock, 1981).

\subsection{Bolometric Luminosity}

This is the luminosity of a galaxy across all wave lengths. It is measured in watts.

A relation between bolometric luminosity and the luminosity of the forbidden line of doubly ionized oxygen [OIII] $\lambda 5007 \AA$ is as follows [7]:

$$
\mathrm{L}_{\text {bol }}=3500 \mathrm{~L}_{[\mathrm{OIII}]}
$$

However, the [OIII] line is susceptible to reddening and as such would be extinction corrected: Following [2],

$$
L_{[O I I I]}=4 \pi d^{2} F_{[O I I I}^{C o r}
$$

$$
\text { But } F_{[O I I I]}^{C o r}=F_{[O I I I]}^{o b s}\left[\frac{\left(\frac{H_{\alpha}}{H_{\beta}}\right)^{o b s}}{\left(\frac{H_{\alpha}}{H_{\beta}}\right)^{0}}\right]^{2.94}
$$

Where $F^{c o r}$ the extinction is corrected flux density, $\mathrm{F}^{\text {obs }}$ is the observed flux density, $\left(\frac{H_{\alpha}}{H_{\beta}}\right)^{0}$ is Balmer decrement with zero reddening usually 2.86 .

Substituting equations (2) and (3) in (1) gives

$$
L_{b o l=14,000} \pi d^{2} F_{[O I I I]}^{o b s}\left[\frac{\left(\frac{H_{\alpha}}{H_{\beta}}\right)^{o b s}}{\left(\frac{H_{\alpha}}{H_{\beta}}\right)^{0}}\right]^{2.94}
$$

Where $\mathrm{d}$ is luminosity distance in parsec.

Equation (4) is the extinction corrected expression that we used in calculating the bolometric luminosity of each of the objects under study.

\subsection{Eddington Luminosity and Eddington Ratio}

The Eddington luminosity of a galaxy is the luminosity of that galaxy at which radiation force from its central region equals the gravitational force responsible for the in falling of matter into its nucleus. At this coercive stalemate state, the blackhole should not be accreting. Since the black hole is not accreting, the virial theorem predicts that half the potential energy of the central region will be converted to radiation while the other half forms its internal energy. Eddington ratio of a galaxy is the ratio of the bolometric luminosity to that of Eddington luminosity of that galaxy. It is a pointer to how energetic the central region is; when it is more than unity, it is super-Eddington accretion and when it is less than unity it is sub-Eddington accretion [3].

We use $\mathrm{L}_{\mathrm{Edd}}=1.27 \times 10^{30} \frac{M_{B H}}{M_{0}}$ watts to calculate Eddington luminosity

and $\frac{L_{b o l}}{L_{E d d}}$ to calculate the Eddington ratio of each of the samples.

Figures 1 and 2 are the histograms of the logarithm of the bolometric luminosities of Seyfert 1 and Seyfert 2 galaxies respectively as an approximate normal distribution with Seyfert 1 having an average bolometric luminosity of $\sim 4 \mathrm{x}$ $10^{43} \mathrm{erg}^{-1}$ and Seyfert $2 \sim 4 \times 10^{44} \mathrm{erg}^{-1}$ in consonance with [6] that the bolometric luminosity of Seyfert galaxies ranges from $4 \times 10^{43} \mathrm{erg}^{-1}$ to $4 \times 10^{45} \mathrm{erg}^{-1}$. This results shows that Seyfert 2 galaxies are more luminous than Seyfert 1 galaxies. 


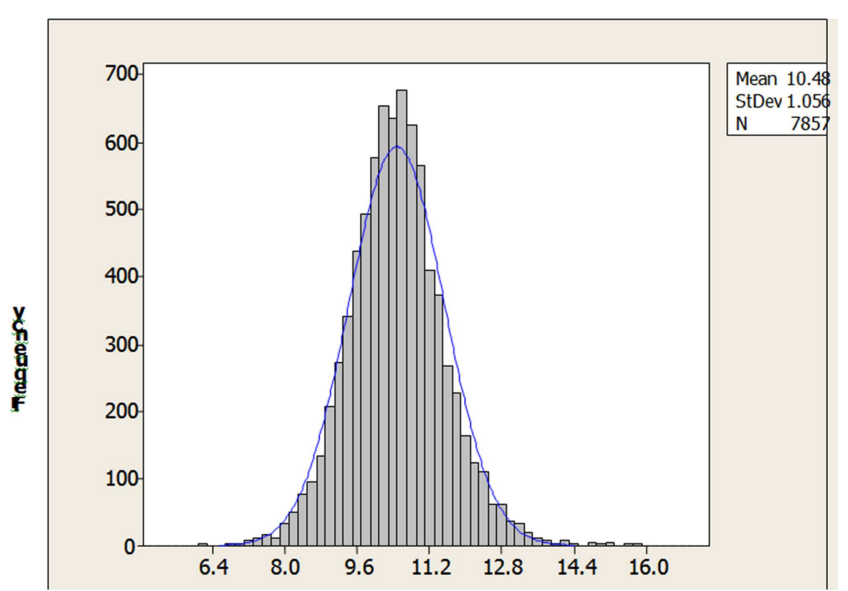

Figure 1. Logarithmic distribution of Bolometric Luminosity for Seyfert 1s.

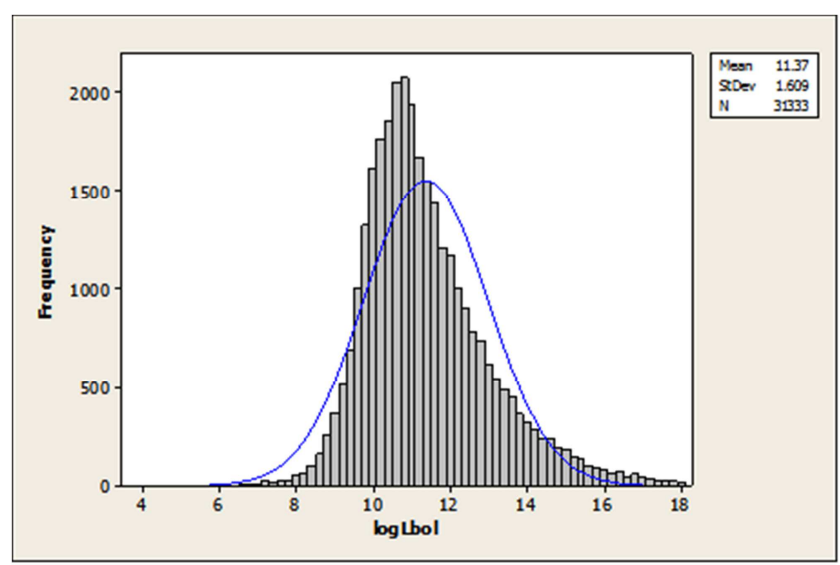

Figure 2. Logarithmic distribution of Bolometric Luminosity for Seyfert $2 s$.

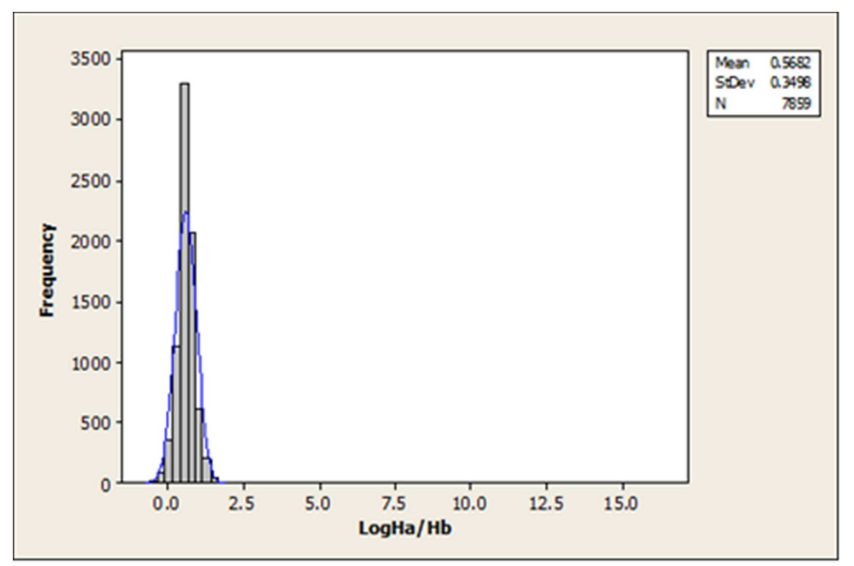

Figure 3. The logarithmic distribution of Balmer decrement for Seyfert 1 s.

Figures 3 and 4 show the logarithm of Balmer decrements for Seyferts 1 and 2 respectively. Mean Balmer decrement in Seyfert 1 is 3.69 with a standard deviation of 0.35 while that of Seyfert $2 \mathrm{~s}$ is 8.79 with a standard deviation of 0.35 . Since reddening is contamination of the Balmer lines by infrared radiations emitted by heated solids, Balmer decrement being higher in Seyfert 2s implies that Seyfert 2 galaxies have more torus than Seyfert $1 \mathrm{~s}$ and this will mean that Seyfert 1 galaxies are younger galaxies and are associated with starburst activities more than Seyfert 2s. However, the reason
Seyfert 2 galaxies have higher bolometric luminosities than Seyfert 1 galaxies is that Seyfert $2 \mathrm{~s}$ have bigger blackhole mass than Seyfert 1 galaxies (figures 5 and 6) and active galactic nuclei activities are based on the nucleus not the constituent stars of a galaxies.

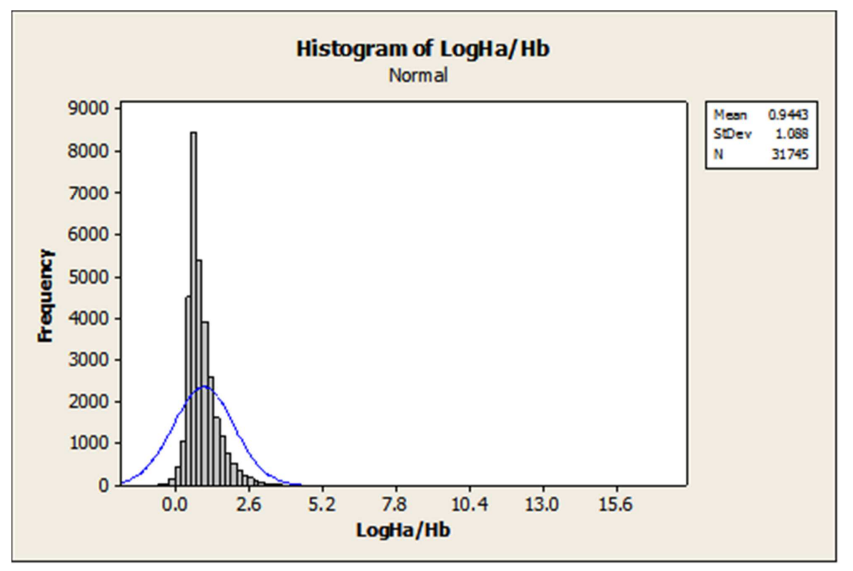

Figure 4. The logarithmic distribution of Balmer decrement for Seyfert $2 s$.

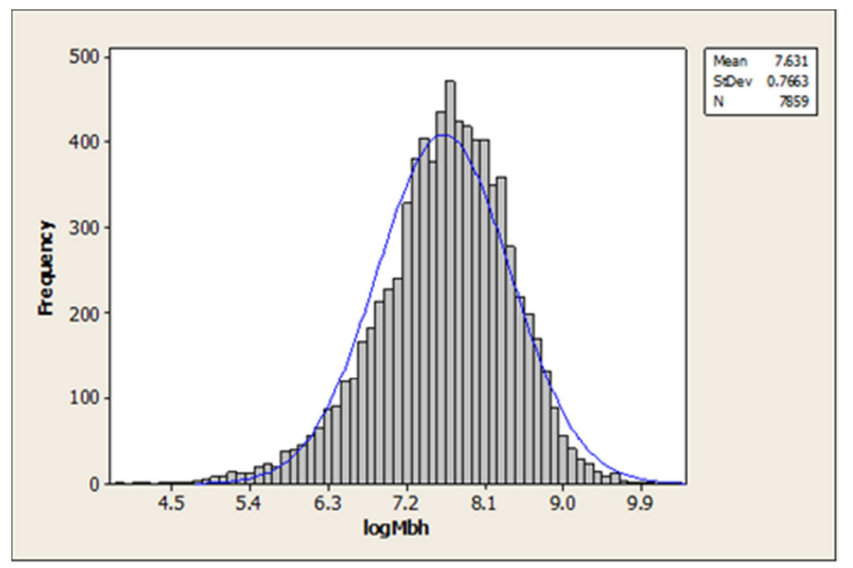

Figure 5. A logarithmic distribution of the blackhole mass of Seyfert 1 galaxies.

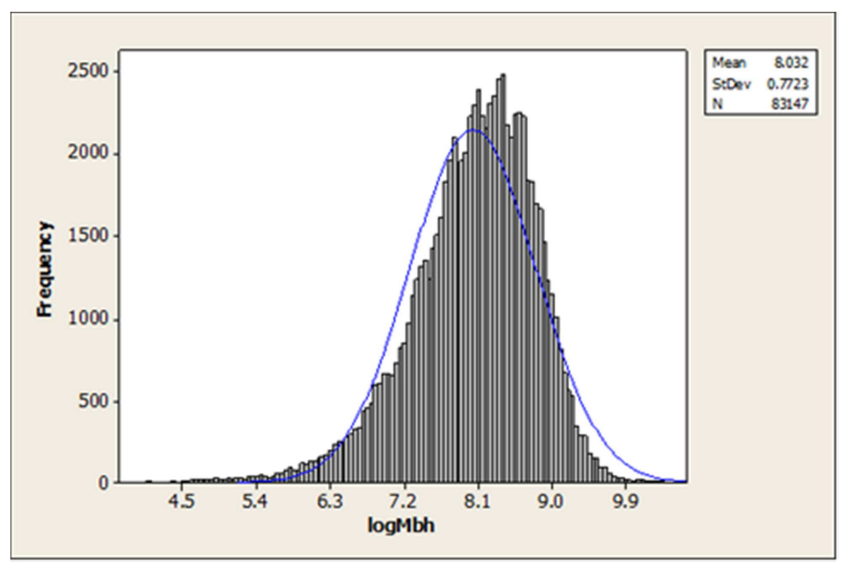

Figure 6. A logarithmic distribution of the blackhole mass of Seyfert 2 galaxies.

Examining the central region of Seyfert galaxies, the logarithmic blackhole mass distribution of both classes of Seyfert galaxies was considered (Figures 5 and 6) and the observation was that seyfert 1 galaxies had a mean black hole 
mass of $10^{7.631} m_{\odot}$ while Seyfert 2 galaxies had a mean black hole mass of $10^{8.032} m_{\odot}$ implying that Seyfert 2 galaxies are centrally more massive than their Seyfert 1 counterparts in agreement with [10].

The logarithmic distribution of Eddington luminosities for Seyferts 1 and 2 are shown in figures 7 and 8 respectively with Seyfert 2 having a mean Eddington luminosity of $10^{7.837}$ $L_{\odot}$ and Seyfert 1 having a mean Eddington luminosity of $10^{7.436} L_{\odot}$ (Figures 7 and 8 ).

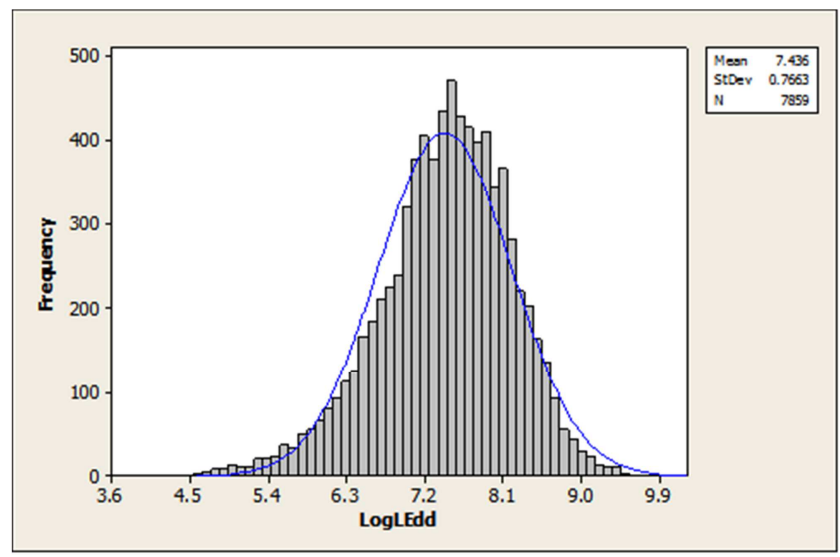

Figure 7. A logarithmic distribution of the Eddington luminosity of Seyfert 1 galaxies.

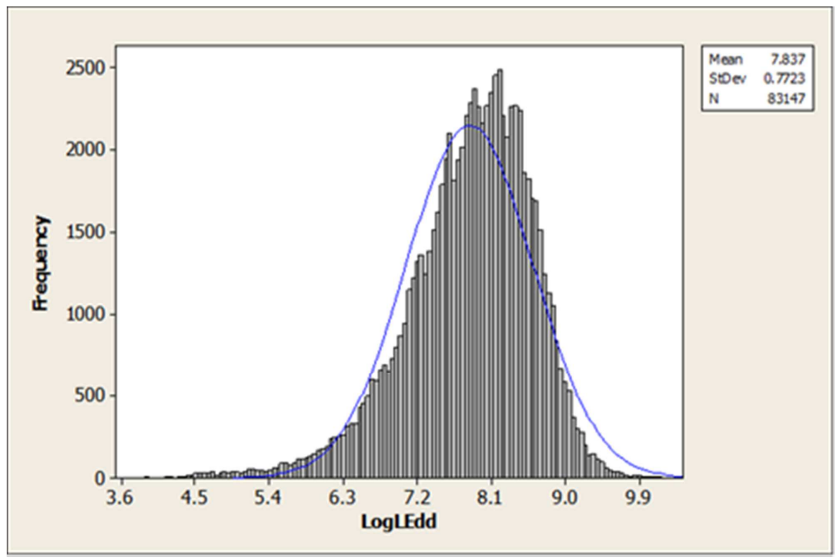

Figure 8. A logarithmic distribution of the Eddington luminosity of Seyfert 2 galaxies.

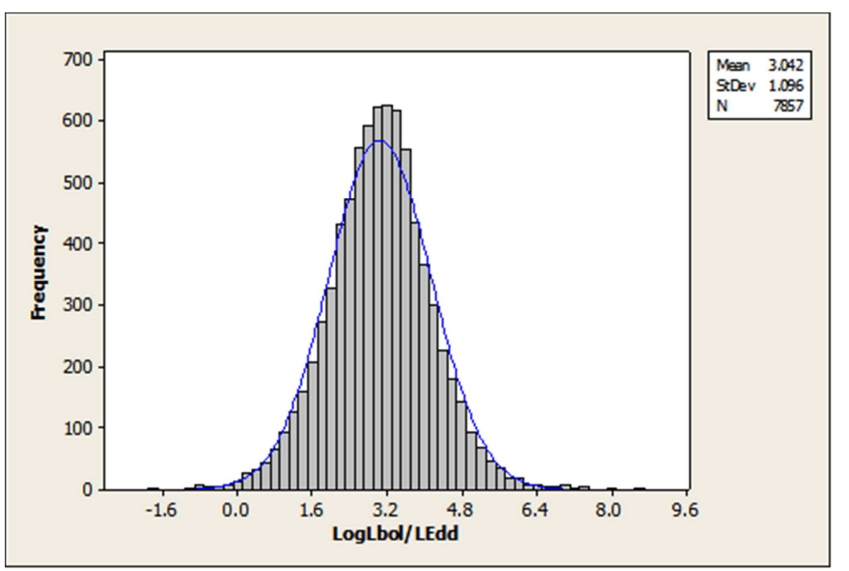

Figure 9. A logarithmic distribution of theEddingtonratios of Seyfert 1 galaxies.

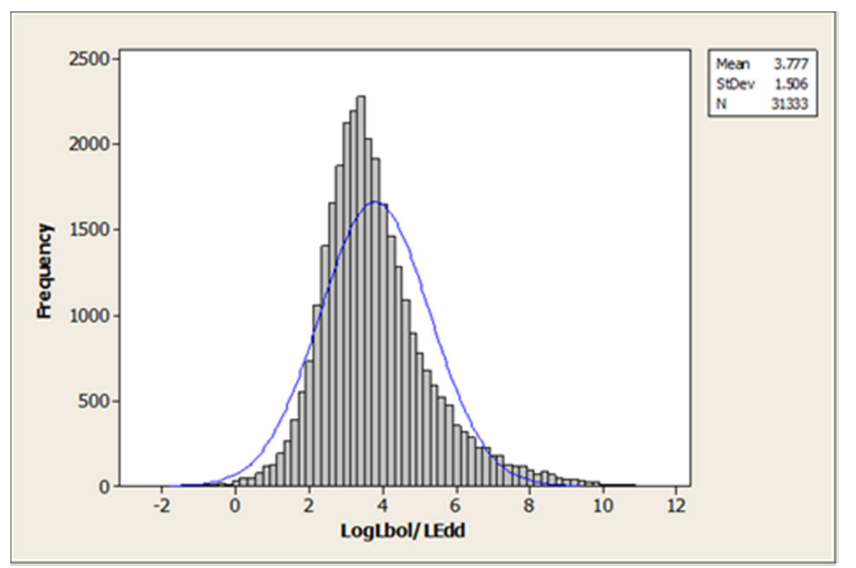

Figure 10. A logarithmic distribution of Eddington ratiosof Seyfert 2 galaxies.

\section{Discussion}

We present the extinction corrected bolometric luminosities of Seyferts 1 and 2 in Figures 1 and 2 as histograms of a normal distribution. Our results yield a mean bolometric luminosity of $L_{b o l} \sim 4 \times 10^{43} \mathrm{ergs}^{-1}$ for Seyfert 1 galaxies and $\sim 4 \times 10^{44} \mathrm{ergs}^{-1}$ for Seyfert 2 galaxies which is consistent with [9] that the bolometric luminosity of Seyfert galaxies ranges from $\sim 4 \times 10^{43} \mathrm{ergs}^{-1}-\sim 4 \times 10^{45} \mathrm{ergs}^{-1}$. Comparing the mean luminosities of both classes of Seyferts shows that the bolometric luminosity of Seyfert 2 galaxies is 10 times that of Seyfet1 galaxies. Looking at the difference in the bolometric luminosities of Seyfert 1 and Seyfert 2 galaxies, this is consistent with [6] who studied galaxy NGC 1068, a barred spiral galaxy and found its bolometric luminosity to be $L_{\mathrm{bol}}=2.4 \times 10^{44} \mathrm{ergs}^{-1}$. This may imply that Seyfert 2 galaxies may be barred Seyfert galaxies since objects of comparable luminosity are likely to have the same nucleus. [3] defined an AGN undergoing super-Eddington accretion as one whose ratio is $\frac{L_{b o l}}{L_{E d d}}>1$, and comparing this with our work, we discovered that of the 7,418 Seyfert 1 galaxies only $36(0.49 \%)$ had Eddington ratios less than 1 and as such are undergoing sub-Eddington accretion with Eddington ratios ranging from 0.0025 to 0.99 . Whereas 7,382 (99.51\%) are undergoing super-Eddington accretion having Eddington ratios greater than unity. For Seyfert 2 galaxies out of 31,333 samples, 31,083 $(99.72 \%)$ are undergoing superEddington accretion while only 88 of them undergo subEddington accretion with Eddington ratios ranging from 0.0014 to $0.99(0.28 \%)$. Our results show that black hole growth in both Seyfert 1 and Seyfert 2 galaxies is dominated by super-Eddington accretion rather than sub-Eddington accretion which is consistent with [8]. Though [4] defined super-Eddington accretion and $\frac{L_{b o l}}{L_{E d d}} \geq 3$ and sub-Eddington accretion as $\frac{L_{b o l}}{L_{E d d}}<3$, our results show that both Seyferts 1and 2 galaxies are powered by thick accretion disc and as such are good accretors (mean Eddington ratio for Seyfert 1 is 3.042 while that of Seyfert 2 is 3.777, (figures 9 and 10). Only a negligible percent of them $(0.49 \%$ for Seyfert 1 galaxies and $0.28 \%$ for Seyfert 2 galaxies) are powered by 
thin accretion disk. Super-Eddington accretion is predominant in both Seyfert 1 and Seyfert 2 galaxies but more in Seyfert $2 \mathrm{~s}$ where Eddington ration is higher making Seyfert 2 galaxies better accretors and centrally more energetic and we conclude that Seyferts of thin accretion disks are in the low-Eddington era.

\section{Conclusion}

In both Seyferts 1 and 2 galaxies, Eddington ratio is greater than unity diagnostically defining both seyfert galaxies as objects undergoing super-Eddington accretion except for a handful of them $(0.49 \%$ for Seyfert $1 \mathrm{~s}$ and $0.28 \%$ for Seyfert $2 \mathrm{~s})$ where sub-Eddington accretion was noticed. In addition, reddening was significantly less in Seyfert 1 galaxies (3.69 in Seyfert 1 and 8.79 in Seyfert 2s) establishing that more infrared radiations are emitted within the central region of seyfert 2 galaxies than seyfert $1 \mathrm{~s}$ which are responsible for the contamination of the Balmer lines emitted from the HII region making them appear red; a condition called reddening.

The sample of galaxies studied in this work was found to have average bolometric luminosity ranging from $10^{43.6}$ $\mathrm{ergs}^{-1}$ (for Seyfert 1s) to $10^{44.6} \mathrm{ergs}^{-1}$ (for Seyfert 2s). We conclude that Seyfert galaxies are objects of predominantly super-Eddington accretion.

\section{References}

[1] Antonucci R. R. J (1993). Unified Models for Active Galactic Nuclei and Quasars ARA and A, 31, 473.

[2] Bassani L, Dadina M, Maiolino R, Salvati M, Risaliti G,
Roberto D.C, Matt G, DellaCeca R., Zamorani G (1999). A three dimensional diagnostic diagram for Seyfert 2 galaxies: Probing X-ray absorption and Comptonthickness. ApJS, 121, 473.

[3] Bian, W., \& Zhao, Y. 2004, MNRAS, 347, 607.

[4] Castello-Mor, N., Kaspi., Netzer. 2017, MNRAS, 467, 1209.

[5] Contini M, Cracco V, Ciroi S, Mura L. A (2012). Distribution of the Heavy Elements Throughout the Extended Narrow-Line Region of the Seyfert Galaxy NGC 7212. Astron. Astrophys. 545: A72. doi: 10.1051/0004-6361/2012191.

[6] Das, V., Crenshaw, D. M., Kraemer, S. B., \& Deo, R. P. 2006, AJ, 132, 620 (paperII). Dopita M. A, Sutherland R. S., 1995, APJ, 455, 468.

[7] Heckman T. M and Best P. N (2014). The Coevolution of Galaxies and Supermassive Blackhole: Insights from surveys of the contemporary Universe, ApJ 613, 109.

[8] Kawaguchi, T., Pieren, A., \& Hure, J.-M. 2004a A\&A, 415, 47.

[9] Meléndez, M., Kraemer, S. B., Armentrout, B. K., RP Deo et al. 2008, ApJ, 682, 94. Osterbrock, D. E., 1981, APJ, 249, 462.

[10] Oyor D. I and Ogwo J. N (2019). On the Extent of the Existence of Broad LineRegion in Seyfert Galaxies; American Journal of Astronomy andAstrophysics. Vol7, No. 2 pp. 33-38. doi: $10.11648 /$ j.ajaa.20190702.12.

[11] Ricci C, Walter R, Courvoisier T. J. L and Paltani S (2011). Reflection in Seyfert Galaxies and the Unified Model of AGN. A \& A 532, A102.

[12] Vaona L., Ciroi S. Dimille F., Cracco V., Mura La G., Rafanelli P., MNRAS (2012). Spectral Properties of the Narrow Line Region in Seyfert Galaxies Selected from SDSS7. Vol. 475 pg. 1266. 